\title{
Anorectal Malformation with Associated Duodenal Obstruction and Uterus Didelphys
}

\author{
MV Vincent ${ }^{1}$, D Madhuri ${ }^{2}$
}

\begin{abstract}
Anorectal malformations have long been associated with multiple other anomalies, commonly referred to as the VACTERL complex. We present a case of a newborn baby girl with an unusually high number of associated anomalies, including the rarely encountered condition of uterus didelphys.
\end{abstract}

Keywords: anorectal malformation, duodenal obstruction, pelvic kidney, uterus didelphys

\section{Malformación anorrectal asociada con obstrucción duodenal y útero didelfo}

\author{
MV Vincent ${ }^{1}$, D Madhuri ${ }^{2}$
}

\begin{abstract}
RESUMEN
Las malformaciones anorrectales se han asociado por mucho tiempo con otras anomalias, conocidas comúnmente como asociación VACTERL. Presentamos el caso de una niña recién nacida con un número inusualmente alto de anomalías asociadas, incluyendo una condición raramente encontrada conocida como útero didelfo.
\end{abstract}

Palabras clave: Malformación anorrectal, obstrucción duodenal, riñón pélvico, útero didelfo

West Indian Med J 2018; 67 (1): 69

\section{INTRODUCTION}

Anorectal malformations (ARMs) present as a disease spectrum which can occur in isolation or in conjunction with other well-described congenital anomalies. We present the case of a newborn baby girl with an ARM and multiple associated anomalies. The associated anomalies included malrotation without volvulus, duodenal atresia (Type 1), absent gallbladder, meckel's diverticulum, a right pelvic kidney, and uterus didelphys.

\section{CASE REPORT}

A newborn baby girl was referred to the surgical unit of The Queen Elizabeth Hospital in Barbados, after her newborn examination revealed an absent anal opening. The baby was born by emergency Caesarean section after her mother presented with meconium-stained liquor with associated fetal decelerations at 40 weeks' gestation. The mother was a 26-year-old primigravida with a history of polycystic ovarian disease. She first presented antenatally at 32 weeks' gestation when her initial antenatal scan revealed the presence of a fetal double bubble, a finding which persisted throughout her pregnancy.

At birth, the baby weighed $2.88 \mathrm{~kg}$ with Apgar scores of 8 at one minute and 9 at five minutes. She was immediately transferred to the neonatal intensive care unit where she required no cardiorespiratory support.
From: 'Department of Surgery, The Queen Elizabeth Hospital, Barbados, West Indies and ${ }^{2}$ Department of Radiology, The Queen Elizabeth Hospital, Barbados, West Indies.
Correspondence: Dr MV Vincent, Department of Surgery, The Queen Elizabeth Hospital, Martindale's Road, Bridgetown, St Michael, Barbados, West Indies. Email: michvincent@yahoo.com 
On examination, three unusual perineal openings were noted. She possessed an anal dimple with the anal orifice located anteriorly (a vestibular fistula), through which copious amounts of meconium was seen to be evacuating. Her vaginal orifice revealed the presence of a midline septum, and a normal urethral opening was noted (Fig. 1). She had no limb deformities or dysmorphic facial features.

A nasogastric tube was passed and placed on free drainage, after which a portable chest X-ray was performed. This revealed the presence of a persistent double bubble as well as the presence of 13 ribs bilaterally (Fig. 2). Further investigations were subsequently ordered, and the following anomalies were revealed.

An echocardiogram (on day 1 of life) showed significant persistent pulmonary hypertension. The right heart chamber was also dilated and a patent foramen ovale present with a small secondary atrial septal defect with left-to-right shunting.

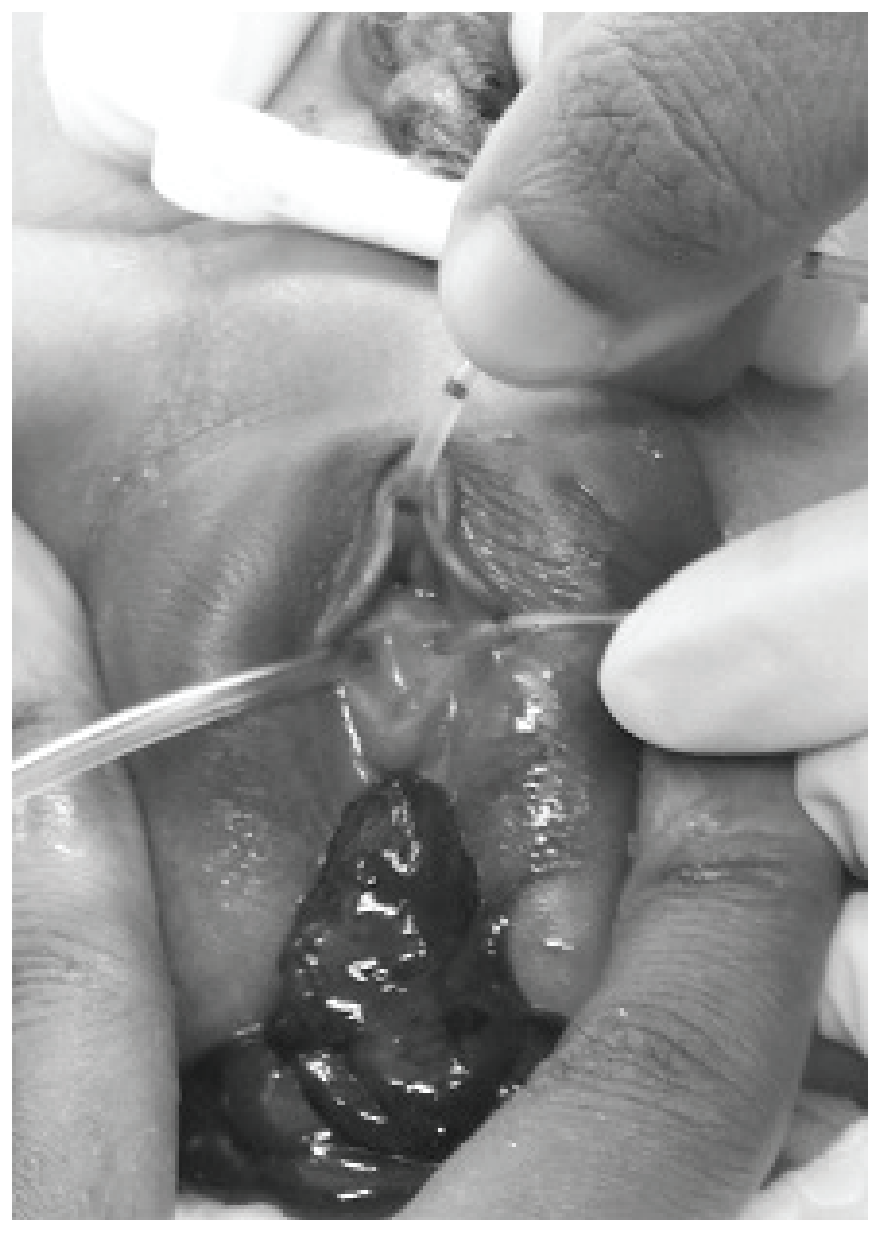

Fig. 1: Perineal openings showing catheters in the urethra and both orifices of the double vagina. Meconium is also noted emanating from the vestibular fistula.
Abdominal and pelvic ultrasound scans revealed a right pelvic kidney and uterus didelphys (duplication of the vagina, cervix and uterus).

On day 3 of life, the baby underwent exploratory laparotomy and the formation of an umbilically-sited distal descending colostomy, under triple-antibiotic cover (ampicillin, gentamycin and flagyl). The protective colostomy was placed in preparation for the second stage of management of her ARM - posterior sagittal anorectoplasty.

Intraoperatively, she was noted to have an absent gallbladder. The proximal duodenum was moderately dilated and bulbous with the presence of an intraluminal membrane. The caecum and appendix were located in the right upper quadrant with the majority of the small bowel located in the right side of the abdomen, and the presence of a meckel's diverticulum measuring only 3 $\mathrm{mm}$ in length. The sigmoid colon and rectum were both distended with copious amounts of meconium.

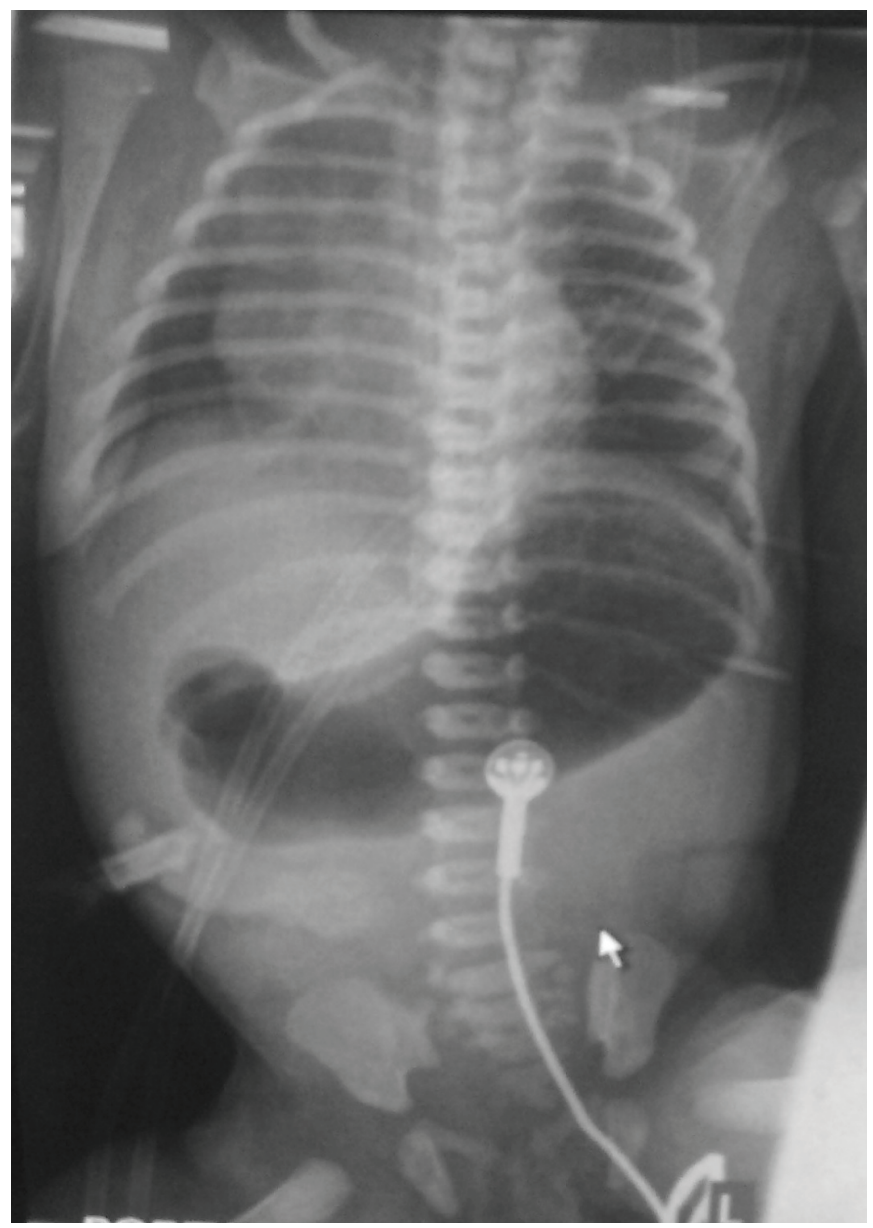

Fig. 2: Radiograph showing 13 ribs bilaterally. The classical 'double bubble' of duodenal obstruction is also apparent. 
A diamond-shaped duodenojejunostomy was performed, together with appendicectomy, and an umbilically-sited distal descending colostomy with the distal end narrowed to a mucus fistula. Prior to the formation of the mucus fistula, the meconium within the rectum and sigmoid colon was emptied and both areas irrigated with saline.

Postoperatively, the baby was stable with the passage of meconium per stoma and moderate quantities of nasogastric aspirates (ranging from 90 to $130 \mathrm{ml}$ per day), which was noted to be mildly to moderately bilious in nature. On day 3 postoperatively, the baby began having bradycardic episodes with associated desaturations, and was initially placed on ventilator continuous positive airway pressure (CPAP) via high flow nasal cannulation and then subsequently intubated and ventilated. Her blood cultures revealed the growth of a gram-negative bacillus (Acinobacter) and so antibiotics were changed initially to vancomycin, gentamycin and flagyl and finally to meropenem, gentamycin and flagyl based on sensitivities. However, the baby remained critically ill despite supportive care and developed generalized oedema, worsening neutropenia and worsening thrombocytopenia. She was also noted to have persistent desaturations while ventilated with evidence of persistent pulmonary hypertension of the newborn. Her urine output tailored, and she developed bloody secretions from the mouth and eventually succumbed to overwhelming sepsis from Acinobacter on day 6 of life.

\section{DISCUSSION}

Anorectal malformations have an estimated incidence of 1 in 5000 live births (1-3). The condition presents in a spectrum ranging from mild anal anomalies to complex cloacal malformations. The aetiology remains unclear, though a genetic component has been proposed, particularly for vestibular fistulas $(1,4,5)$. In fact, patients with perineal or vestibular ARMs are two to three times more likely to have a family member with an ARM, and this is important with respect to genetic counselling $(1,4)$.

It has now been over 40 years since ARMs have been known to be associated with a variety of anomalies, commonly known as the VACTERL association, which comprises vertebral anomalies, anal atresia, cardiac malformations, trachea-oesophageal fistula, renal anomalies and limb abnormalities $(1,5,6)$.

Genitourinary complications can occur in up to $50 \%$ of patients with an ARM $(2,4)$. In girls with a vestibular ARM, gynaecological anomalies occur in about $17 \%$ $(4,7)$, while those with more complex ARMs such as cloacal anomalies have an even higher incidence of $53-67 \%(2,3,7-11)$. These gynaecological anomalies include hemivagina, vaginal septum, vaginal atresia/ absent vagina, multiple vaginas, absent cervix, absent uterus, unicornuate uterus, bicornuate uterus, uterus didelphys, and duplication of the genitalia. Many of these gynaecological anomalies may be missed until adolescence or early adulthood, especially if no attempt is made to exclude them during the neonatal period. These include:

- vaginal septum (symptomatic in almost half of adult patients) which can be associated with the prevention of effective use of a tampon for menstrual flow, painful vaginal intercourse, cyclic pelvic pain, abnormal vaginal bleeding, bleeding during intercourse and an intermittent mucopurulent discharge $(7,8,12)$;

- hemivagina which can lead to hydrocolpos with associated hydroureteronephrosis, haematocolpos, haematometra with the risk of endometriosis, and pelvic inflammation $(8,9,11,12)$;

- uterine anomalies (unicornuate uterus, bicornuate uterus, uterus didephys) leading to an increased risk of preterm labour, preterm delivery and abnormal fetal presentations; and

- absent cervix leading to abnormal menstrual flow and infertility $(7,13)$.

As noted in this case, uterus didelphys consists of the presence of two uterine horns each with a cervix (14-16). This rare congenital anomaly is thought to arise from the failure of lateral fusion of the mullerian ducts and hence is often associated with ipsilateral renal anomalies, most commonly renal agenesis $(12,15-19)$.

If the anomaly is associated with separate vaginas, as in this case, the girl is usually asymptomatic as there is an outlet for physiological and menstrual secretions (9). However, an asymmetric bicornuate uterus with a rudimentary horn does not communicate with the vagina and will usually become symptomatic with the onset of menstruation. Common presentations include lower abdominal pain, a tender suprapubic mass, urinary retention and constipation $(9,11,14-16)$. In such cases, there is cyclic bleeding from the hemi-uterus and vagina of the unobstructed side with obstructive symptoms and development of haematometra from the contralateral obstructed uterus $(9,16)$.

Thus, it has been proposed that all girls should undergo vaginoscopy at the time of posterior sagittal anorectoplasty (PSARP) or at the time of the formation 
of a protective colostomy, with vaginoscopy and cystoscopy \pm laparoscopy being performed in those with more complex ARMs $(2,7,8,11)$. This is to ensure timely diagnosis of associated gynaecological anomalies, assist with surgical planning, and prevent the above-mentioned complications at puberty, with its associated long-term sequelae $(2,7,9,11,12,16)$. It is also generally thought that performing these procedures during the neonatal period will prevent the associated psychological trauma of undergoing 'vaginal surgery' during later childhood or adolescence $(7,8)$.

Girls with vestibular ARMs and an isolated vaginal septum can be effectively managed at the time of PSARP. A window or defect can be made within the septum to allow drainage of both hemivaginas, or the septum can be completely resected $(7,8,16)$. Resection of the vaginal septum is most commonly performed using a scalpel or scissors. Other less invasive techniques include hysteroscopic resection, with the preservation of the hymen (18), or resection using combined laparoscopy and vaginoscopy (16).

In summary, ARMs in girls can be associated with a variety of genitourinary anomalies. Knowledge of these associations is important so that early surgical interventions can be provided and serious complications such as chronic pelvic inflammation and infertility can be minimized. Long-term follow-up into adulthood must also be provided as inevitably the growing patient and parents will have concerns with respect to future sexual intimacy and reproductive potential $(8,11)$.

\section{REFERENCES}

1. Falcone Jr RA, Levitt MA, Pena A, Bates M. Increased heritability of certain types of anorectal malformations. J Pediatr Surg 2007; 42: 124-8.

2. Pandya KA, Koga H, Okawada M, Coran AG, Yamataka A, Teitelbaum DH. Vaginal anomalies and atresia associated with imperforate anus: diagnosis and surgical management. J Pediatr Surg 2015; 50: 431-7.

3. Shukla VK, Mishra A, Shukla RC, Pandey S, Pandey LK, Gangopadhyay AN. An extremely unusual presentation of high anorectal malformation: rectovaginal fistula with sacral agenesis and uterus didelphys. J Indian Assoc Pediatr Surg 2004; 9: 151-4.
4. Herman RS, Teitelbaum DH. Anorectal malformations. Clin Perinatol 2012; 39: 403-22.

5. Solomon BD, Raam MS, Pineda-Alvarez DE. Analysis of genitourinary anomalies in patients with VACTERL (vertebral anomalies, anal atresia, cardiac malformations, trachea-esophageal fistula, renal anomalies, limb abnormalities) association. Congenit Anom (Kyoto) 2011; 51: 87-91.

6. Quan L, Smith DW. The VATER association. Vertebral defects, anal atresia, T-E fistula with esophageal atresia, radial and renal dysplasia: a spectrum of associated defects. J Pediatr 1973; 82: 104-7.

7. Levitt MA, Bischoff A, Breech L, Pena A. Rectovestibular fistula rarely recognized associated gynaecologic anomalies. J Pediatr Surg 2009; 44: 1261-7.

8. Breech L. Gynecological concerns in patients with anorectal malformations. Seminars in Pediatric Surgery 2010; 19: 139-45.

9. Nayci A, Avlan D, Oz U, Toktas S, Aksoyek S. Does menstrual flow exclude hematometra? A rare case of uterine anomaly presenting with anorectal malformation. J Pediatr Surg 2002; 37: 666-7.

10. Levitt MA, Stein DM, Pena A. Rectovestibular fistula with absent vagina: a unique anorectal malformation. J Pediatr Surg 1998; 33: 986-90.

11. Chadha R, Puri M, Saxena R, Agarwala S, Puri A, Choudhury R. Congenital pouch colon in a girl associated with bilateral atresia of the cervix uteri and uterus didelphys. J Indian Assoc Pediatr Surg 2013; 18: $81-3$.

12. DaCosta V, Christie L, Wynter S, Harriott J, Frederick J. Uterus bicornis bicollis, imperforate hemivagina and ipsilateral renal agenesis: case report and literature review. West Indian Med J 2009; 58: 379-82.

13. Fujimoto VY, Miller JH, Klein NA, Soules MR. Congenital cervical atresia: report of seven cases and review of the literature. Am J Obstet Gynecol 1997; 177: 1419-25.

14. Pansini L, Torricelli M, Gomarasca A, Brambilia C, Beolchi S, Sideri M. Acute urinary retention due to didelphys uterus associated with an obstructed hemivagina in a 5-month-old infant. J Pediatr Surg 1988; 23: 984-5.

15. Skondras KG, Moutsouris CC, Vaos GC, Barouchas LD, Demetriou LD. Uterus didelphys with an obstructed hemivagina and ipsilateral renal agenesis: a rare cause of acute abdomen in pubertal girls. J Pediatr Surg 1991; 26: 1200-1.

16. Patterson D, Mueller C, Strubel N, Rivera R, Ginsburg HB, Nadler EP. Laparoscopic neo-os creation in an adolescent with uterus didelphys and obstructed hemivagina. J Pediatr Surg 2006; 41: E19-22.

17. Wu TH, Wu TT, Ng YY, Ng SC, Su PH, Chen JY, Chen SJ. HerlynWerner-Wunderlich syndrome consisting of uterine didelphys, obstructed hemivagina and ipsilateral renal agenesis in a newborn. Pediatr Neonatol 2012; 53: 68-71.

18. Tsai EM, Chiang PH, Hsu SC, Su JH, Lee JN. Hysteroscopic resection of vaginal septum in an adolescent virgin with obstructed hemivagina. Hum Reprod 1998; 13: 1500-1.

19. Ghouloum S, Puligandla PS, Hui T, Su W, Quiros E, Laberge J. Management and outcome of patients with combined vaginal septum, bifid uterus, ipsilateral renal agenesis (Herlyn-Werner-Wunderlich syndrome). J Pediatr Surg 2006; 41: 987-92. 